\title{
NEUROFIBROMATOSE PLEXIFORME RETROPERITONEAL E PÉLVICA: ASPECTOS NA TOMOGRAFIA COMPUTADORIZADA E RESSONÂNCIA MAGNÉTICA - RELATO DE CASO E REVISÃO DA LITERATURA*
}

\author{
Daniel Dutra Cançado ${ }^{1}$, Camila Cruz Leijoto ${ }^{2}$, Carlos Eduardo Souza Carvalho ${ }^{3}$, Paulo Roberto \\ Valle Bahia ${ }^{4}$
}

Resumo Os autores apresentam um caso de neurofibromatose tipo I, caracterizado por múltiplos neurofibromas plexiformes no retroperitônio e pelve. A tomografia computadorizada mostrou lesões marcadamente hipoatenuantes, acometendo principalmente psoas e regiões paravertebrais, aspectos que mimetizavam outras doenças, como abscessos e linfonodomegalias. A ressonância magnética foi útil para a melhor caracterização da extensão dos neurofibromas, bem como de sua estrutura interna.

Palavras-chave: Neurofibromatose; Plexiforme; Tomografia computadorizada; Ressonância magnética.

Abstract Retroperitoneal and pelvic plexiform neurofibromatosis: computed tomography and magnetic resonance findings - case report and review of literature.

The authors report a case of neurofibromatosis type I, characterized by multiple plexiform neurofibromas in the retroperitoneum and pelvis. Computed tomography showed markedly hypodense lesions involving mainly the psoas and the paravertebral regions, findings which could mimic other diseases such as abscesses and lymphadenopathy. Magnetic resonance imaging was useful to better evaluate the extension and internal structure of the neurofibromas.

Key words: Neurofibromas; Plexiform; Computed tomography; Magnetic resonance imaging.

\section{INTRODUÇÃO}

A neurofibromatose tipo 1 (NF-1), ou doença de von Recklinghausen, é doença de caráter autossômico dominante, sendo a mais comum das facomatoses (síndromes neurocutâneas), ocorrendo em um a cada 2.000 nascimentos. A NF-1 é caracterizada principalmente por manchas do tipo cafécom-leite, múltiplos neurofibromas periféricos, sardas nas axilas, gliomas ópticos, hamartomas de íris e lesões ósseas ${ }^{(1)}$.

Os neurofibromas plexiformes são lesões que praticamente definem o diagnóstico de NF-1 e consistem em massas múl-

* Trabalho realizado nos Departamentos de Radiodiagnóstico Reumatologia e Anatomia Patológica do Hospital Universitário Clementino Fraga Filho (HUCFF) da Universidade Federal do Rio de Janeiro (UFRJ), Rio de Janeiro, RJ.

1. Médico Residente do Departamento de Radiologia da UFR J

2. Médica Residente do Departamento de Reumatologia da UFR.

3. Médico do Departamento de Anatomia Patológica da UFRJ. 4. Professor Assistente do Departamento de Radiologia da UFR.

Endereço para correspondência: Dr. Daniel Dutra Cançado. Rua Jornalista Henrique Cordeiro, 120, bl. 2, ap. 1703, Barra da Tijuca. Rio de Janeiro, RJ, 22631-450. E-mail: danieldutra@ wnetrj.com.br

Recebido para publicação em24/6/2004. Aceito, após revisão, em 13/8/2004. tiplas, tortuosas, vermiformes, que crescem ao longo do eixo de um grande nervo, infiltrando e separando os fascículos nervosos normais, produzindo uma aparência fusiforme $e^{(2)}$.

O objetivo deste trabalho é apresentar um caso de neurofibromatose plexiforme do retroperitônio e da pelve, seus achados de imagem na tomografia computadorizada (TC) e ressonância magnética (RM), bem como uma discussão do tema baseado na literatura.

\section{RELATO DO CASO}

Paciente de 26 anos de idade, sexo feminino, inicialmente internada para investigação de quadro reumatológico. Há cerca de um ano a paciente vinha apresentando poliartralgia assimétrica e migratória em punhos, ombros e joelho esquerdo, além de lombalgia e cervicalgia, com piora progressiva dos sintomas ao longo desse período. Havia sinais de artrite no joelho esquerdo, com derrame articular.

Havia relato de emagrecimento $(8 \mathrm{~kg} \mathrm{em}$ um ano), alopécia e surgimento de man- chas do tipo café-com-leite. A paciente relatava também a excisão de dois nódulos cutâneos na coxa direita, com o diagnóstico de neurofibroma.

Exame de artrocentese do joelho esquerdo foi negativa para artrite inflamatória/infecciosa.

Devido a uma queixa de dor abdominal difusa e massa palpável em mesogástrio, foi realizada ultra-sonografia abdominal, que revelou múltiplos nódulos hipoecogênicos no retroperitônio, distribuídos bilateralmente, localizados anteriormente ao músculo psoas e adjacentes à aorta e veia cava inferior, cuja impressão inicial foi de um conglomerado linfonodal.

A TC abdominal demonstrou lesões bem delimitadas, marcadamente hipoatenuantes, com fraca impregnação pelo meio de contraste, localizadas na região paravertebral, acometendo preferencialmente o músculo psoas, estendendo-se para os flancos e para a musculatura do ilíaco e paravertebral (Figura 1). A TC mostrava também a insinuação das lesões em direção aos forames neurais, aspecto que foi melhor avaliado na RM com cortes coronais. A 


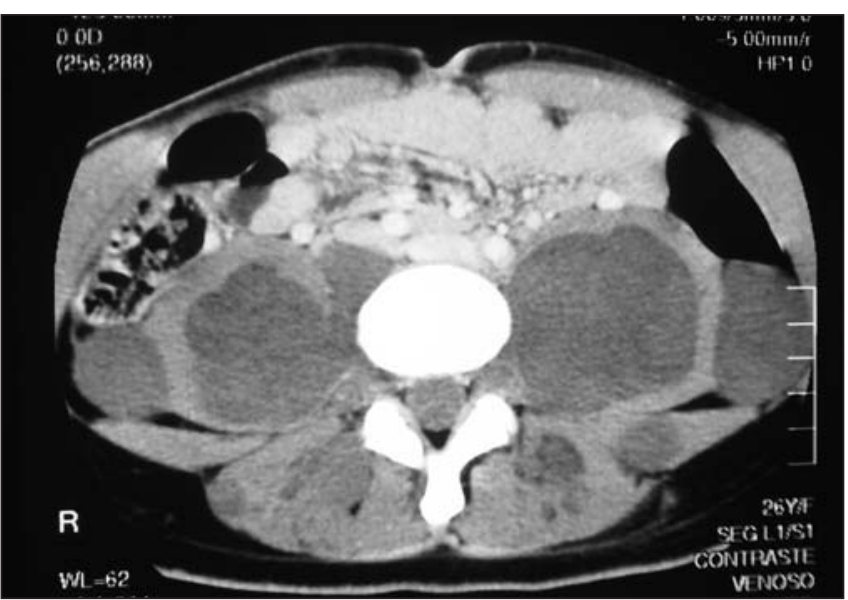

A

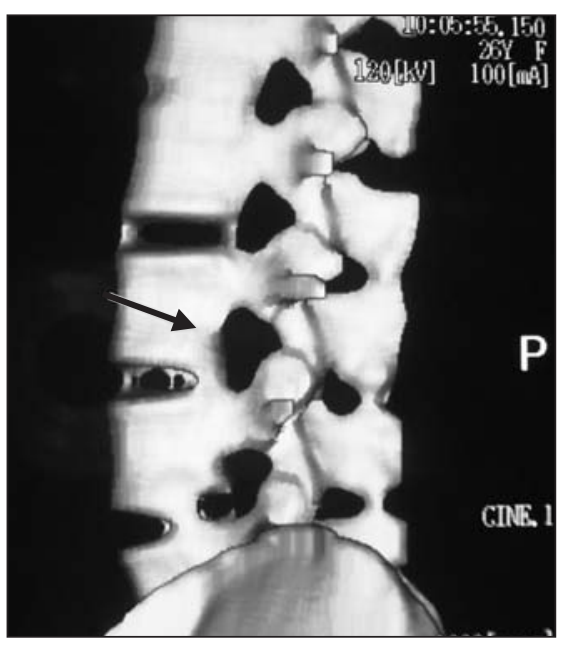

C

reconstrução 3D de superfície da coluna vertebral mostrou alargamento do forame neural de L3-L4.

$\mathrm{Na} \mathrm{RM}$, as lesões mostraram-se isointensas e homogêneas na ponderação T1 e hiperintensas na ponderação T2, com pequenas áreas com hipossinal no interior dessas lesões (Figura 2). Apesar do estudo por RM não ter sido direcionado especificamente para a coluna vertebral, não havia evidências de envolvimento do canal raquiano.

A paciente foi submetida a biópsia percutânea guiada por TC, a qual, associada aos aspectos da imagem, confirmou o diagnóstico de neurofibroma plexiforme.

\section{DISCUSSÃO}

Os neurofibromas plexiformes são uma marca registrada da NF-1, ocorrendo em cerca de um terço dos pacientes. Eles dide L3-L4 (seta).

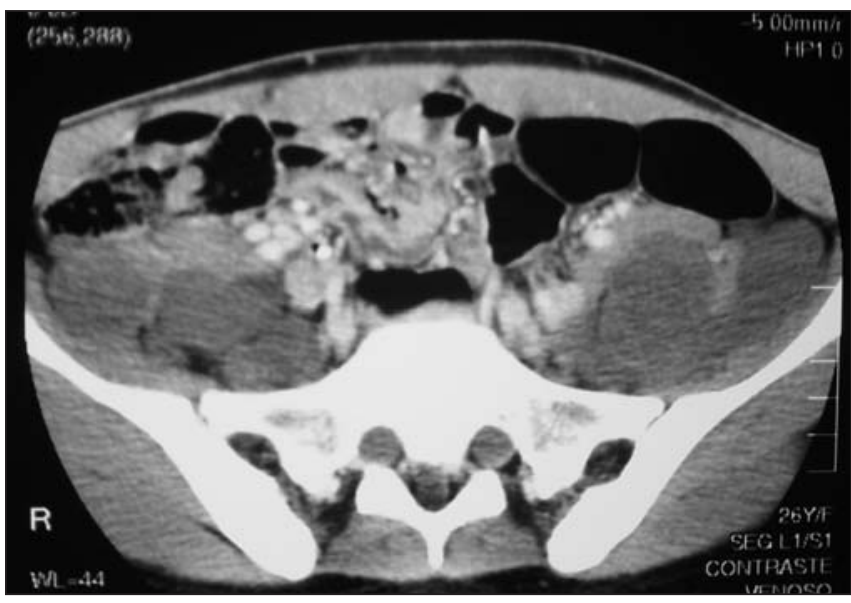

Figura 1. Tomografia computadorizada de abdome evidenciando múltiplas lesões hipodensas, com fraca impregnação pelo meio de contraste, localizadas na musculatura do psoas, nos flancos, na pelve e musculatura paravertebral $(\mathbf{A}, \mathbf{B})$. A reconstrução 3D da coluna lombar $(\mathbf{C})$ mostra alargamento do forame neural

ferem dos neurofibromas cutâneos, em parte devido à grande quantidade de matriz extracelular ${ }^{(3)}$. As lesões consistem em proliferação de células na bainha nervosa estendendo-se ao longo do nervo e envolvendo múltiplos fascículos. Freqüentemente, há envolvimento de uma porção significativa de um nervo principal, bem como suas ramificações, produzindo massa difusa de nervos espessados. É importante ressaltar que o termo "plexiforme" não implica o envolvimento de um plexo nervoso, como, por exemplo, o plexo lombar ou braquial, apesar dessas regiões serem também acometidas ${ }^{(4)}$.

Os neurofibromas plexiformes podem estar confinados nos tecidos moles profundos ou podem envolver a pele e a musculatura, neste último caso produzindo hipertrofia e distorção dos tecidos adjacentes, com conseqüente deformidade. Outra complicação desses tumores é a transformação maligna; cerca de $10 \%$ dos pacientes com NF-1 desenvolvem tumores malignos da bainha nervosa, que geralmente se originam de um neurofibroma plexiforme pré-existente $^{(\mathbf{3 , 5})}$.

$\mathrm{Na}$ tomografia, os neurofibromas se apresentam como lesões arredondadas, com contornos regulares e margens distintas. Eles apresentam valores baixos de atenuação (20-25 UH) e reforço homogêneo pelo meio de contraste, com valores de atenuação de 30-50 UH. Esta baixa atenua- ção é atribuída principalmente a células de Schwann ricas em lipídios e produção de matriz extracelular ${ }^{(\mathbf{6})}$. O achado de lesões extensas, hipoatenuantes e de caráter infiltrativo, por vezes na topografia de feixes nervosos, sugerem a presença de neurofibromas plexiformes ${ }^{(\mathbf{1 , 7}, \mathbf{8})}$.

Devido às diversas formas de apresentação da NF-1 — no tórax, abdome, pelve e extremidades —, os neurofibromas podem simular uma série de lesões na TC, tais como linfonodomegalias, abscessos, outros tumores neurogênicos, metástases, meningoceles ou sarcomas de partes moles. Nestes casos, é necessária a biópsia para o correto diagnóstico ${ }^{(\mathbf{1})}$.

Em casos de neurofibromatose plexiforme retroperitoneal, os achados de imagem na TC podem sugerir o diagnóstico. Bass et al. ${ }^{(9)}$ estudaram 16 pacientes com neurofibromatose plexiforme retroperitoneal e observaram que, em 15 deles, a TC mostrava achados semelhantes: massas hipoatenuantes, bilaterais e simétricas, estendendo-se ao longo dos músculos psoas e região pré-sacral. $\mathrm{O}$ estudo sugeria que, na presença desses achados de imagem em pacientes com NF-1, a biópsia seria desnecessária para a confirmação de neurofibromatose plexiforme. A biópsia destas lesões, além de acarretar riscos inerentes ao procedimento invasivo, pode resultar em déficits neurológicos, já que neurônios funcionantes estão presentes dentro do tumor. 

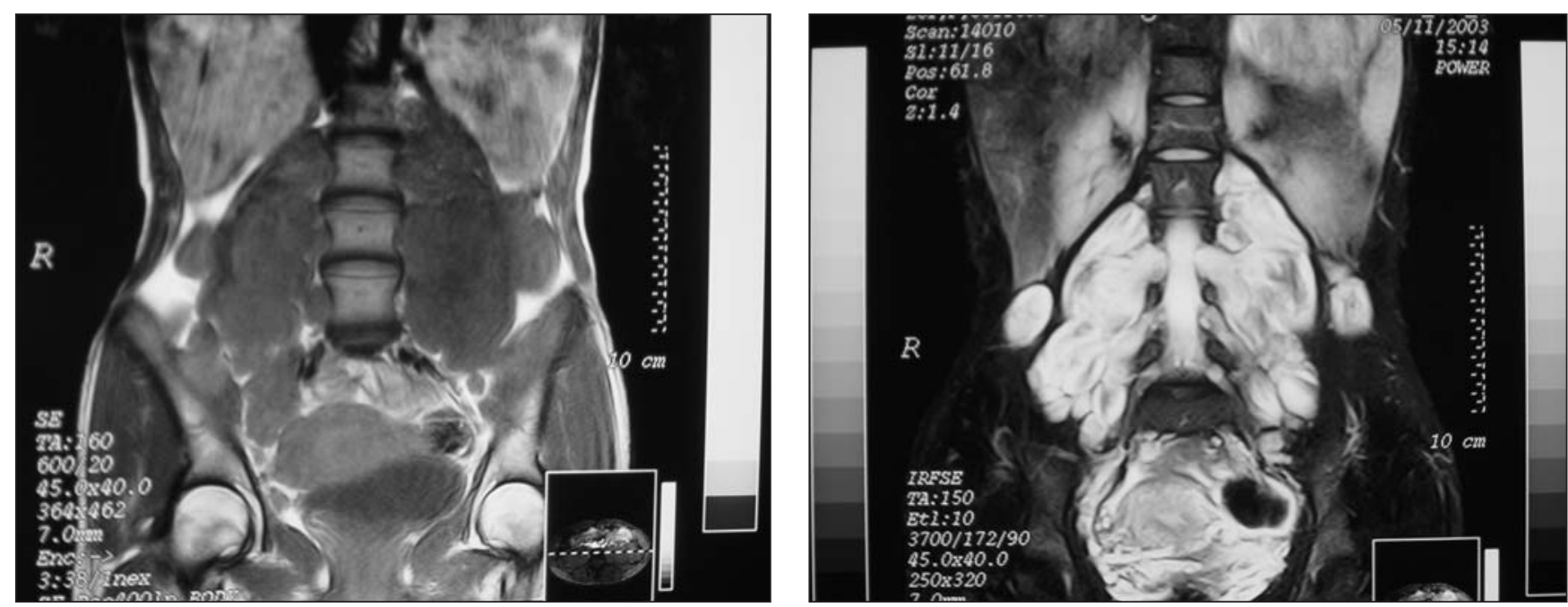

A

Figura 2. Ressonância magnética do abdome nas seqüências ponderadas em T1 e T2 no plano coronal $(\mathbf{A}, \mathbf{B})$ e axial (C). Os neurofibromas apresentamse homogêneos e isointensos em relação à músculatura na ponderação T1 (A) e discretamente heterogêneos na ponderação T2, predominando o hipersinal com algumas septações hipointensas no interior das lesões (B,C). Observa-se a insinuação das lesões em direção aos forames neurais (B) e sua extensão até a região pélvica $(\mathbf{A}, \mathbf{C})$.

Os aspectos de imagem das lesões encontradas em nosso caso são comparáveis com os achados deste estudo.

A RM tem a capacidade de demonstrar melhor a natureza interna do neurofibroma, bem como de outros tumores da bainha nervosa, quando comparada à TC. Por sua capacidade multiplanar, a RM fornece melhor avaliação da extensão das lesões, principalmente dos neurofibromas plexiformes ${ }^{(10)}$. Os neurofibromas exibem sinal de intermediário a levemente hiperintenso na ponderação $\mathrm{T} 1$, em relação ao músculo. Na ponderação T2, as lesões são marcadamente hiperintensas, devido à presença de matriz mixóide ${ }^{(\mathbf{1 0 , 1 1})}$. A RM pode também revelar septações hiperintensas na ponderação T1, pela presença de axônios mielinizados, e septações hipointensas na ponderação T2, estas últimas relacionadas à presença de fibras colágenas dentro do tumor ${ }^{(\mathbf{1 0 )}}$. A RM também pode mostrar atrofia da musculatura inervada pelo nervo acometido. Este achado, associado a uma tumoração na topografia de um nervo principal, sugere tumor de bainha nervosa ${ }^{(11)}$.

A principal complicação do neurofibroma plexiforme é a transformação maligna,

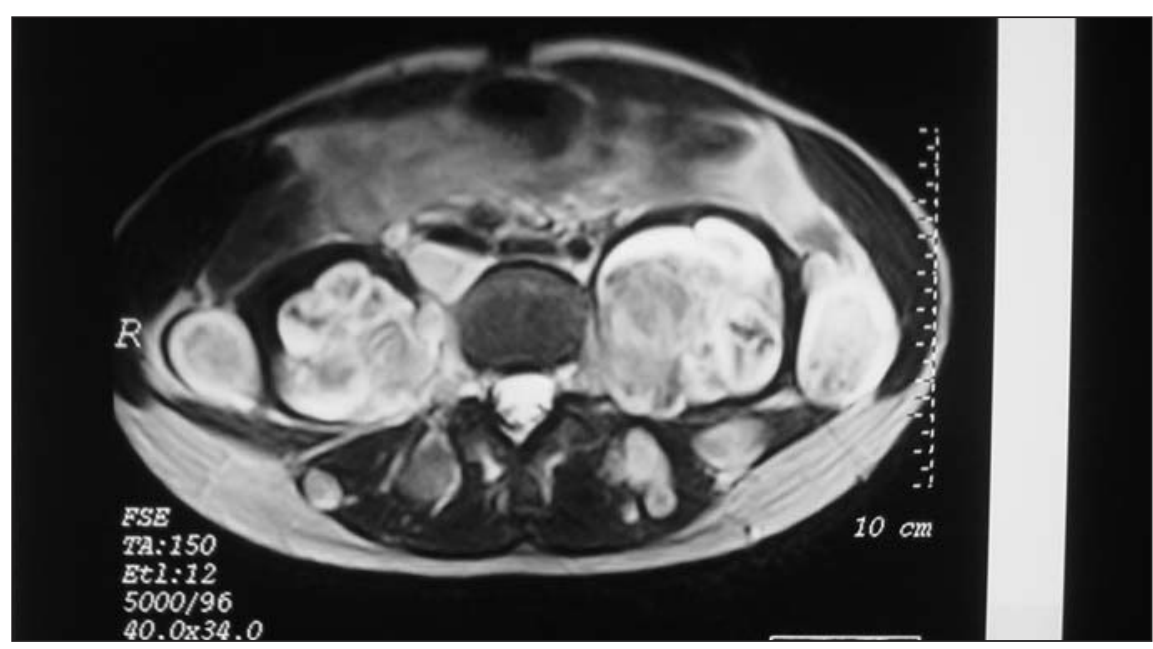

C

como já mencionado. Os sinais clínicos suspeitos de degeneração maligna incluem surgimento de dor persistente, déficit neurológico e aumento da massa tumoral ${ }^{(\mathbf{4 , 5})}$.

O uso da TC e RM para a diferenciação de neurofibromas benignos dos tumores malignos de bainha nervosa tem apresentado resultados controversos na literatura, em boa parte devido à pequena casuística e a algumas limitações técnicas, como o uso de aparelhos de RM de baixo campo, ausência de técnicas de saturação de gordura e a não injeção endovenosa de meio de contraste em alguns estudos ${ }^{(5)}$.

No estudo de Bass et al. ${ }^{(9)}$, sobre neurofibromas plexiformes retroperitoneais, foi observado que o crescimento assimétrico de uma das massas retroperitoneais, associado a um aumento da sua atenuação, era indicativo de transformação maligna.
Levine et al. ${ }^{(\mathbf{1 2})}$ e Stull et al..$^{(11)}$, analisando as margens tumorais e a heterogeneidade das lesões, não conseguiram distinguir com clareza - pela TC ou pela RM a natureza benigna ou maligna das lesões. Já Mautner et al. ${ }^{(\mathbf{5})}$, ao estudarem 50 pacientes com neurofibromatose plexiforme submetidos à RM com contraste, observaram que nos dez pacientes que apresentaram lesões heterogêneas — seja por áreas de necrose, hemorragia ou reforço heterogêneo pelo meio de contraste - foi confirmada a presença de tumor maligno de bainha nervosa.

Outros métodos diagnósticos, como a cintilografia com ${ }^{67} \mathrm{Ga}$ e mais recentemente o ${ }^{18}$ FDG PET (tomografia por emissão de pósitrons), mostraram-se promissores na identificação das lesões com degeneração maligna $^{(\mathbf{1 2}, 13)}$. 
Concluindo, a neurofibromatose plexiforme deve ser incluída no diagnóstico diferencial de lesões retroperitoneais com baixa atenuação na TC, principalmente se forem extensas e bilaterais. Contudo, é importante afastar doenças mais freqüentes do retroperitônio, como abscessos e linfonodomegalias, que podem apresentar o mesmo aspecto de imagem na TC.

A RM é útil para o diagnóstico diferencial, pois caracteriza melhor a natureza interna dos neurofibromas plexiformes, e, devido à aquisição multiplanar, define com maior clareza a extensão das lesões.

\section{REFERÊNCIAS}

1. Fortman BJ, Kuszyk BS, Urban BA, Fishman EK Neurofibromatosis type 1: a diagnostic mimicker at CT. RadioGraphics 2001;21:601-12.
2. Osborn AG. Distúrbios da histogênese: síndromes neurocutâneas. In: Osborn AG, ed. Diagnóstico neurorradiológico. Rio de Janeiro: Revinter, 1999: $72-7$.

3. Tonsgard JH, Kwak SM, Short MP, Dachman AH. CT imaging in adults with neurofibromatosis-1: frequent asymptomatic plexiform lesions. Neurology 1998;50:1755-60.

4. Korf BR. Plexiform neurofibromas. Am J Med Genet 1999;89:31-7.

5. Mautner VF, Friedrich RE, von Deimling A, et al. Malignant peripheral nerve sheath tumours in neurofibromatosis type 1: MRI supports the diagnosis of malignant plexiform neurofibroma. Neuroradiology 2003;45:618-25.

6. Rha SE, Byun JY, Jung SE, et al. Neurogenic tumors in the abdomen: tumor types and imaging characteristics. RadioGraphics 2003;23:29-43.

7. Fenton LZ, Foreman N, Wyatt-Ashmead J. Diffuse, retroperitoneal mesenteric and intrahepatic periportal plexiform neurofibroma in a 5-year-old boy. Pediatr Radiol 2001;31:637-9.

8. Gossios KJ, Guy RL. Case report: imaging of wide- spread plexiform neurofibromatosis. Clin Radiol 1993;47:211-3.

9. Bass JC, Korobkin M, Francis IR, Ellis JH, Cohan RH. Retroperitoneal plexiform neurofibromas: CT findings. AJR 1994;163:617-20.

10. Ros PR, Eshaghi N. Plexiform neurofibroma of the pelvis: CT and MRI findings. Magn Reson Imaging 1991;9:463-5.

11. Stull MA, Moser RP Jr, Kransdorf MJ, Bogumill GP, Nelson MC. Magnetic resonance appearance of peripheral nerve sheath tumors. Skeletal Radiol 1991;20:9-14.

12. Levine E, Huntrakoon M, Wetzel LH. Malignant nerve-sheath neoplasms in neurofibromatosis: distinction from benign tumors by using imaging techniques. AJR 1987;149:1059-64.

13. Ferner RE, Lucas JD, O'Doherty MJ, et al. Evaluation of (18)fluorodeoxyglucose positron emission tomography ((18)FDG PET) in the detection of malignant peripheral nerve sheath tumours arising from within plexiform neurofibromas in neurofibromatosis 1. J Neurol Neurosurg Psychiatry 2000; 68:353-7. 